\title{
Evaluating Operational Risk Exposure Using Fuzzy Number Approach to Scenario Analysis
}

\author{
Antonina Durfee ${ }^{1}$ Alexey Tselykh ${ }^{2}$ \\ ${ }^{1}$ Citizens Bank, Royal Bank of Scotland Group, Rhode Island, USA \\ ${ }^{2}$ Department of Applied Informatics, Taganrog Institute of Technology, Southern Federal University, Russia
}

\begin{abstract}
Risks and losses arising from system failure, unauthorized activity, fraud and other operational errors are postulated to be one of the primary banking risks. Expert-based scenario analysis aims at describing the frequency and severity of extreme operational losses. Experts are not exact in predicting quantitative estimates for a distant future. The predicted scenarios are described by range estimates and qualitative storylines. This study introduces and applies the methodology for evaluation of the operational risk levels derived from the experts opinions collected from scenarios. The methodology is based on the use of fuzzy numbers to express subjective probability of expert estimates.
\end{abstract}

Keywords: operational risk, scenario analysis, fuzzy decision making, fuzzy number

\section{Introduction}

In the light of the global financial crisis, both media, leading policy makers, economists and practitioners are questioning the appropriate levels of risk in the international financial system. Decision makers and banking practitioners are struggling to evaluate the risk attributed to the globalization of modern banking, the fast evolving information technology, new economic theories, opaque financial instruments, and complex mathematical modeling. International banking industry had been trying to balance the existing risks in the financial systems for quite some time by regulating the approaches to measure and manage risks. Although the latest financial crisis made us question some regulatory requirements, it had accentuated the necessity to evaluate risks more holistically.

The Bank for International Settlements, an international organization which fosters international monetary and financial cooperation and serves as a bank for central banks, had realized that developing banking practices such as securitization, outsourcing, specialized processing operations and reliance on technology had introduced substantial risks which need to be reflected in credible capital assessments. As a result, Basel II Accord that includes risks other than credit and market was proposed to be "a comprehensive framework for improving bank safety and soundness by more closely linking regulatory capital requirements with bank risk, by improving the ability of supervisors and financial markets to assess capital adequacy", according to Fed- eral Reserve Chairman Ben S. Bernanke in a May 2006 speech at the Federal Reserve Bank of Chicago's 42nd Annual Conference on Bank Structure and Competition. All the largest banks in the world that carry international component are mandated to become Basel certified. Resulting international risk management practice for financial institutions focuses on three main risk categories: Market Risk, Credit Risk and Operational Risk (OR). OR is a relatively newly defined category of risk which includes many different types of risk, from the simple operations risks of transaction processing, unauthorized activities and systems risks to other types of risk that are not included in market or credit risks, such as human, legal, informational and reputational risks with the exception of strategic risk [2]. By contrast with credit and market risks where acceptable models and measurements exist, it is relatively difficult to identify and evaluate levels of operational risk due to the complexity of its origin, lack of existing historic quantitative data and explanation. One of the best publicized OR risk events are rouge trading in the French Société Générale bank, BP oil spill, and American Maddoff "ponzi" scheme causing billions of dollars to a global financial system.

Scenario analysis as a decision making tool has been in existence for several decades and has been used in various disciplines, including management, engineering, defense, medicine, finance and economics [6]. Scenario analysis is one of the four required data elements that banks must incorporate into their Advanced Modeling Approach to adequately measure their operational risk levels. Scenario analysis brings a forward looking element into OR assessment since it describes future plausible severe OR loss events. The current range of practice published in 2009 by Basel Committee of Banking Supervision [16] identifies a lack of consistent controls to address scenario analysis bias noting that scenario analysis is subjective in nature. Chosen and estimated scenarios become an effective technique for stimulating management to identify key strategic operational risks facing their businesses. Scenario workshops are used as the preferred method to develop a set of scenarios. During scenario workshop, experts are supplied with a library of plausible extreme operational events that have or may have happened in peer financial organization and are asked to assess the likelihood and severity of similar events if they occur in their business, as described by the Bank of Japan [25]. Scenarios as a framework offer the ability to bring qualitative business intuition data into a systematic assessment of OR which is increasingly important in an era of stress testing of international financial sector. Making point estimates in 
a distant future is cognitively prohibitive for humans which form their judgment better as vague linguistic or in-range estimates.

This paper presents a methodology based on fuzzy approach for estimation of the operation risk level from a set of plausible scenarios. There are two main contributions of this paper. The first one is of the most importance to the practitioner. It lies in the proposal of the systematic approach to integrate scenario data which is "fuzzy" and vague in nature into OR quantification system. The second contribution is more important for theoretician since it extends the theory and applicability of fuzzy numbers.

The paper starts with the brief description of the current state of affairs in terms of the usage of scenarios in OR management. Then we describe the applicability of fuzzy approaches for decision making. This paper follows with a proposition of the research problem and the description of research methodology. It continues with an example of research methodology applicability and description of validation study which are very important when a new method is introduced. The paper concludes with discussion on limitations and future work.

\section{Literature Overview}

The literature review provides definition of operational risk and description of existing standards for measuring OR. It provides relevant insight into explanation of scenarios and their use as a mandatory data element in evaluation of OR. The theory of fuzzy numbers and their use in decision making, problem solving in general and OR in particularly is provided.

\subsection{Operational Risk under Basel II}

Decision makers are weighting their alternative in terms of their risk-benefit composition. Risk can be defined as an exposure to uncertainty, according to [8]. Risk is subjective in nature, because different people may have different views on the uncertainty. Moreover, the same person might have dissimilar views on the same risk after assessing it from another point of view. Subjective nature of risk may bring a decision maker to a different conclusion evoking different actions.

The term "operational risk" can be viewed as a catchall for financial risks that are not traditionally classified as market or credit risks. Under Basel requirement for risk measurement and capital levels, operational risk is described as a risk not covered by credit or market risks which lie in operational controls and business environment of a bank. Operational losses arise from failure of controls classified in one of seven Basel event categories: External Fraud, Internal Fraud, Execution and Delivery Management, Damage to Physical Assets, Client Practice and Business Process, Employee and Workplace, Business Disruption and System Failure.

Basel II and various Supervisory bodies of the countries have prescribed soundness standards for OR management for banks and similar financial institutions, which should follow one of the three accepted approaches:
- Basic Indicator Approach - based on annual revenue of the Financial Institution,

- Standardized Approach - based on annual revenue of each of the broad business lines of the Financial Institution,

- Advanced Measurement Approaches (AMA) based on the internally developed risk measurement framework of the bank.

Under AMA, a bank must have a comprehensive operational risk quantification system, using inputs from its internal loss data and assessment systems, that provides an estimate of the bank's operational risk exposure, which is defined as the 99.9th percentile of the distribution of potential aggregate operational losses over a one-year horizon. The bank's operational risk exposure is the starting point in determining the riskbased capital requirement for operational risk, according to Basel II guidance document. The 99.9th percentile can be roughly adequate to the one in 1000 year loss. Under Basel framework, operational risk regulatory capital needs to be building on four data elements:

- bank's internal loss experience - where actuarial loss distribution approach is usually employed,

- external/industry wide loss experience - where actuarial or Bayesian approaches are employed,

- scenario analysis - where ad-hoc approaches are still emerging,

- business environment and control factors.

In order to compute adequate regulatory capital which offsets operational losses, a bank's operational risk quantification system must use a combination of internal operational loss event data, relevant external operational loss event data, business environment and internal control factor assessments, and scenario analysis results. The bank should combine these elements in a manner that most effectively enables it to quantify its operational risk exposure. Bank has to build appropriate analytical framework which best describes its business model. An observed loss experience stored as internal loss data (ILD) creates a foundation for the framework and is used in loss distribution approach (LDA). LDA is an actuarial method where severity and frequency of occurred loss are modeled using statistical distribution fitting method. In LDA losses are considered to be identically and independently distributed variables. The assumption used in LDA are limiting since some of the losses might not yet be observed by an institution and some can be not independent and identically distributed.

In order to overcome the limitation posed by ILD and LDA, Basel regulation requires the use of data produced via scenario analysis.

\subsection{Scenario Analysis as a Mandatory Data Ele- ment in Measuring Operational Risk Level and a Decision Making Tool}

Scenario analysis, according to the NPR Supervisory Guidance, is "a systematic process of obtaining expert opinions from business and risk management experts to derive reasoned assessments of the likelihood and loss impact of plausible high-severity operational losses". 
Scenario analysis is especially relevant for business lines or operational loss event types in which internal data, external data, or assessments of business environment and internal control factors do not provide a sufficiently robust estimate of the bank's exposure to operational risk. For example, a bank's scenario analysis should include consideration of high-severity loss events that occur infrequently in the industry. It could also include the effects of mergers or other significant organizational changes that may affect the nature of operational losses in the future. Business line and risk management experts' use of well-reasoned, external data may itself be a form of scenario analysis.

The broad use of the term "scenario" to characterize the systematic framing of uncertain possibilities can be traced to post-World War II strategic studies when Kahn and Wiener [10] explored possible consequences of nuclear proliferation, defining scenarios as "hypothetical sequences of events constructed with the purpose of focusing attention on causal processes and decision point". Most organizations according to Ringland [19] use a Pierre Wack Intuitive Logics in their scenario framework. This approach was first introduced by former Shell group planner Pierre Wack and is concentrated on "creating a coherent and credible set of stories of the future as a "wind tunnel" for testing business plans or projects, prompting public debate or increasing coherence". Initiated and developed in the 1960s and 1970 s, it aims at finding ways of dealing with mindsets of managers so that they can anticipate various futures and prepare for them $[7,26]$. The long term goal is to improve scenario analysis for strategic decision making.

There is a distinction between quantitative (modeling) and qualitative (narrative) traditions of scenario analysis. Quantitative analysis often relies on formal models, using mathematical algorithms and relationships which represent key features of human and environmental systems, such as interest rate which is "stressed". Quantitative modeling is often used for predictive analysis, which is appropriate for simulating well-understood systems such as engineering over short times. But as complexity increases and the time horizon of interest lengthens, the power of prediction diminishes [9]. For a long time, the degree of the state of the system under consideration cannot be specified, the dynamics governing change are not fully understood, and precise mathematical algorithms cannot be created. In such case, even probabilistic forecasting of a given future state, or a spectrum of possible states, becomes not feasible lowering the applicability of simulations and quantitative scenarios.

The limitations of quantitative analysis mean that it should be complemented by qualitative scenario exploration to capture other factors influencing the future states. The scenario storylines describe the important qualitative factors shaping future decisions. Approaches described in [20, 24, 25] combine the long-term narratives with scenarios quantification. From a methodological point of view, scenario authors can attempt to discern the likely outcome of a range of "expected" trends, outline the implications of different assumptions not chosen on the basis of likelihood (what-if analysis) or examine the feasibility and implications of desirable futures - or risks of undesirable ones [24].

Scenario analysis in OR in banking is designed to inform bank executives in terms of bank financial exposure in case of plausible rare extreme OR loss event. Events, such as Madoff ponzi scheme or hurricane Katrina, that have very low probability of happening but high severity and thus require large capital to cover the loss for a bank to stay liquid.

Unlike other data components in AMA, scenario analysis does not have a well-accepted theoretical quantification approach. One of the approaches in the literature tries to combine by bootstrapping, internal loss data in a form of distribution with distribution of losses derived in scenario analysis [6]. The approach relies on well statistically defined frequency and severity distributions. It assumes that the same shapes of distribution can be extended by severity data but not change. However a dramatic event, such as "black swan" analogy described by Taleb in his "The Black Swan", can potentially change the shape and parameters of fit distributions. Another approach in account for scenario data in quantification of OR is to use scenario analysis for derivation of priors in Bayesian quantification method [22]. This approach is difficult to implement since the scenario participants are not able to predict distribution shapes and parameters for priors.

Scenario analysis can be viewed as an extraction of mental models from the knowledge workers. The mental models incorporate intuition which requires sophisticated data mining methods.

\subsection{Related Literature: Fuzzy Approach to Mea- suring Operational Risk Level}

The methodology of this paper is built on the fuzzy number definition provided in [5] by Dubois and Prade, on the $\alpha$-cut concept [11] as well as on the concept of fuzzy probability presented in [4].

This paper does not aim at extensive overview of the literature on fuzzy sets [27] and theory but rather at exploration of its applicability to evaluation of OR from scenario derived expert opinions. The idea of fuzziness and general hypothetical applicability of fuzzy logic to OR domain was given in [23]. In [14, 21] experts based on their knowledge and experience provide if-then fuzzy rules that relate loss amounts to key risk indicators. The paper on "Fuzzy Capital Budgeting" by Kuchta [12] that evaluated fuzzy cash flows was an inspiration of the applicability of fuzzy numbers to scenario description in operational risk.

\section{Research Problem}

Expert opinion is solicited for description of a potential large and plausible extreme operation risk loss event. A group of subject matter experts are asked to describe extreme plausible OR event in a workshop-like meeting to minimize overconfidence and anchoring biases. Subject matter experts are chosen as the ones who have extensive business experience, know-how, and intuition. Prior to the workshop they are supplied with relevant 
descriptive information about large OR events observed in the industry, internal and external operational loss events which provide unbiased ground for their multicriteria decision making in terms of choosing the most likely scenario story line and producing frequency and financial impact estimates. The workshops assemble groups of business line and risk management experts. The teams are asked to review samples of operational loss scenarios sourced from collected external industry data on operation events and predict the likelihood of such an event occurring at the host company and, given that it occurs, the likely financial impact on the firm. An example can be an occurrence of rogue trading the level of SocGen at Citigroup.

A set of bank's subject matter experts are then asked the following questions:

- what type of nearly catastrophic events are plausible to ever happened to their business granted business profile, historic loss experience of the bank and loss experience of the banks with similar business profiles,

- what will be the financial impact of the discussed scenario loss event,

- how often this scenario might happen.

Teams are asked to produce optimistic, realistic, and pessimistic forecasts for each scenario storylines which produce three severity and frequency estimates.

Based on the all inclusive universe of completed scenarios, a decision maker, a person responsible for setting up adequate level of capital, has the following question that needs to be answered:

If all of the scenarios happen, what will be bank exposure and how much capital a bank needs to put aside in order to cover the losses resulting from those scenarios and remain liquid?

Scenario data is derived via structured round table discussion in terms of estimation of plausibility and financial severity of various loss events among experts. There could be any number of scenarios in a complete data set. The requirement is to have a set of scenarios that holistically describes all possible and known OR dangers. The number of scenario data points can be a function of the number of business activities a financial institution is participating in and Basel loss event types. The sample of typical data derived via workshop discussion is presented below in Table 1 . In a course of a workshop, for instance, on external fraud in retail brokerage, a circle of experts comes up with optimistic, realistic, and pessimistic story lines - a set of hypothetical conditions upon which the best, average, and worstcase financial impact can occur. Financial severity or impact from the scenario is measured in dollars and is presented as min, mode, and max severity (or optimistic, realistic, and pessimistic). Experts make their predictions of likelihood in terms of how many years will lapse between this scenario events occurring again. The frequency of a scenario is given in years. There are three frequency estimates that the experts are asked to provide: max, mod, and min frequency. For instance, scenario 108 can happen at the maximum once in 15 years (least frequently), on average (most probable) once every ten years or at the minimum once every 5 years (most frequently). In order to help expert frame their imagination, 100-year time horizon is used.

There is a confidence estimate (in percentages) that experts provide at the workshop which informs us how confident experts are in the completeness of their estimates in terms of severity and frequency of a plausible scenario event. This estimate informs decision maker in the perception of completeness of information experts had at the time of the workshop. It answers the question whether experts had all the correct financial knowledge and right people at the table to come up with frequency and financial impact estimates.

As one can see, both frequency and severity estimates can be presented as triangular fuzzy numbers [5, 13]. The question is how to formally answer a question posted by decision maker mathematically using fuzzy logic.

\section{Research Methodology: Computing Regulatory Capital Based on Scenario Data Using Fuzzy Numbers and Subjective Probabilities}

Pessimistic, realistic, and optimistic expert judgments $\left[a_{\min }, a_{\bmod }, a_{\max }\right]$ on financial impact of scenario can be naturally represented by a triangular fuzzy number $A$ $=\left(a_{\min }, a_{\bmod }, a_{\max }\right)$.

In order to perform fuzzy arithmetic operations [11] with fuzzy numbers we transform to LR-type triangular fuzzy number $A_{L R}=\left(a, \gamma_{A}, \delta_{A}\right)$, where $a=a_{\mathrm{mod}}$, $\gamma_{A}=a-a_{\min }, \delta_{A}=a_{\max }-a$ (Fig. 1).

In a similar manner we can represent expert judgments $\left[b_{\min }, b_{\text {mod }}, b_{\max }\right]$ about the frequency of scenario as a triangular fuzzy number. According to Basel II regulations [3], a risk horizon for evaluation of operational risk is set at one year. Consequently, if the regulatory capital has to cover the possible losses that the entity may suffer from all the OR dangers within oneyear period, the annual frequency should be modeled. Hence, we proceed to a subjective (fuzzy) probability $P\left(A_{i}\right)$ of suffering a financial loss $A_{i}$ :

$$
P\left(A_{i}\right)=\left(1 / b_{\max }, 1 / b_{\bmod }, 1 / b_{\min }\right),
$$

or, using LR-type notation,

where

$$
P\left(A_{i}\right)_{L R}=\left(p, \gamma_{P}, \delta_{P}\right),
$$

$$
p=1 / b_{\bmod }, \gamma_{P}=p-1 / b_{\max }, \delta_{P}=1 / b_{\min }-p .
$$

We use the formula for multiplication of triangular fuzzy numbers to calculate an operational risk exposure from the $i_{t h}$ scenario:

$$
\begin{aligned}
& A_{i} \cdot P\left(A_{i}\right) \cong\left(a_{i} p_{i}, a_{i} \gamma_{P}+p_{i} \gamma_{A}-\gamma_{A} \gamma_{P},\right. \\
& \left.a_{i} \delta_{P}+p_{i} \delta_{A}+\delta_{A} \delta_{P}\right) .
\end{aligned}
$$


The degree of confidence (level of presumption) with which an expert makes a judgment corresponds to a notion of $\alpha$-cut of a fuzzy number. The confidence represents the completeness of information that an expert perceives to have at the time of making estimation in terms of severity and frequency of a plausible scenario event. All scenarios in a set are considered to be independent. The entire set is collected to be all encompassing, which means it describes all the known possible events.

Let $A_{i}=\left(a_{i}, \gamma_{i}, \delta_{i}\right)$ be an operational risk exposure from the $i_{\text {th }}$ scenario given by a triangular fuzzy number. We take $\alpha$-cut as follows (Fig. 1):

$$
\begin{aligned}
& O \operatorname{ORC}_{i}=\left[A_{i}\right]^{\alpha}=\left[a-(1-\alpha) \gamma_{i},\right. \\
& \left.a+(1-\alpha) \delta_{i}\right], \forall \alpha \in[0,1] .
\end{aligned}
$$

To calculate an operational risk exposure from two or more scenarios, we add $\alpha$-cuts as fuzzy confidence intervals:

$$
\begin{aligned}
& {\left[\sum_{i=1}^{n} O R C_{i}\right]^{\alpha}=\left[\sum_{i=1}^{n} a_{i}-(1-\alpha) \sum_{i=1}^{n} \gamma_{i}\right.} \\
& \left.\sum_{i=1}^{n} a_{i}+(1-\alpha) \sum_{i=1}^{n} \delta_{i}\right] .
\end{aligned}
$$

We note that it is not always possible to use this formula in practice as the degree of confidence varies from scenario to scenario.

Hence, we consider $\alpha$-cut as trapezoidal fuzzy interval $M_{i}$ represented with a group of five $M_{i}=\left(m_{i}, \overline{m_{i}}, \beta_{i}, \chi_{i}, h_{i}\right)$ with lower bound $m_{i}$, upper bound $\overline{m_{i}}$, left width $\beta_{i}$, right width $\chi_{i}$, and height $h_{i}$ (Fig. 2).

The sum $M_{i}+M_{j}$ of two fuzzy trapezoidal intervals $M_{i}, M_{j}$ is also a trapezoidal fuzzy interval $(\underline{m}, \bar{m}, \beta, \chi, h)$, where

$$
\begin{gathered}
h=\min \left(h_{i}, h_{j}\right), \\
\chi=h\left(\frac{\chi_{i}}{h_{i}}+\frac{\chi_{j}}{h_{j}}\right), \\
\beta=h\left(\frac{\beta_{i}}{h_{i}}+\frac{\beta_{j}}{h_{j}}\right), \\
\bar{m}=\overline{m_{i}}+\overline{m_{j}}+\chi_{i}+\chi_{j}-\chi, \\
\underline{m}=\underline{m_{i}}+\underline{m}-\beta_{i}-\beta_{j}+\beta .
\end{gathered}
$$

When calculating a total regulatory capital required offsetting OR for one year if this year is assumed to be the worst year, i.e. all scenarios are materializing, we divide the intervals describing the scenarios into groups with equal heights. The groups are arranged in the order of decreasing heights. Furthermore, we combine the results of fuzzy interval addition in each of the groups, starting with the group with the maximum height. When combining fuzzy intervals with equal heights the formulae are simplified to

$$
\begin{gathered}
\chi=\chi_{i}+\chi_{j}, \\
\beta=\beta_{i}+\beta_{j}, \\
\bar{m}=\overline{m_{i}}+\overline{m_{j}}, \\
\underline{m}=\underline{m_{i}}+\underline{m_{j}}
\end{gathered}
$$

As a result, we get a trapezoidal fuzzy interval corresponding to optimistic (liberal) and pessimistic (conservative) viewpoints, respectively.

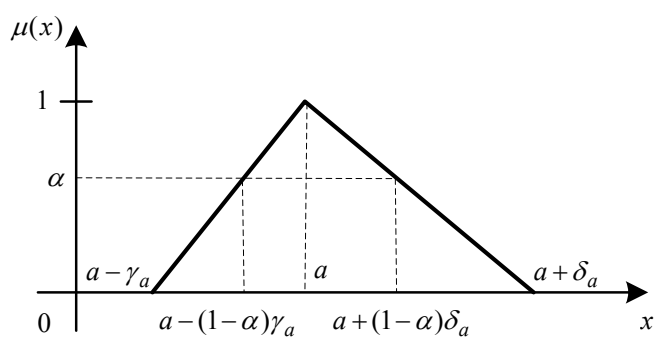

Fig. 1: LR-type triangular fuzzy number

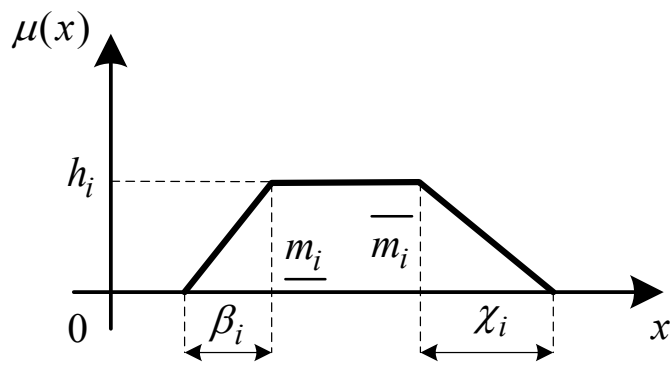

Fig. 2: Trapezoidal fuzzy interval

\section{Results and Analysis}

This section starts with an example of the applicability of the proposed methodology. It continues with the description of real life experiment undertaken as a validation part.

Suppose, there are two hypothetical scenarios: 108 and 114. The scenario 108 comes from the group of the scenarios on External Fraud, which consists of stories describing raids, theft and robberies. In retail banking, branch robberies happen quite often and usually have low financial impact and are considered to be a cost of doing business. As scenario participants predict, in the best set of circumstances, the theft event from scenario 108 can have an impact of $\$ 1.2 \mathrm{mln}$ and has a potential to occur once every 5 years (or 20 times in a course of 100 years). These minimum estimates are framed via an optimistic scenario story line. The same event most probably can cause $\$ 1.7 \mathrm{mln}$ loss but happen once every 10 years (or 10 times in a course of 100 years) framed by realistic storyline with more likely circumstances. At 
the worst case with congruence of bad circumstances which can happen less frequently, - once in 15 years (or 6.7 times in a course of 100 years) - the event 108 would cause the most dramatic loss of $\$ 2.2 \mathrm{mln}$. Similar logic but different estimates are derived by experts for scenario 114 on the breaches of regulatory requirements. The scenario 114 in general is a little less frequent than scenario 108 but more severe. Regulatory breaches can potentially cause large fines and costly law suits. Both scenarios come with alpha estimates which deliver to an analyst an assessment on how complete was the information at hand when experts were making their predictions. The scenario data is presented in Table 1. Estimates adjusted to one year time horizons and given as LR-type triangular fuzzy numbers are presented in Fig. 3 and Fig. 4 respectively.

After applying fuzzy arithmetic, the result from the summation of scenarios 108 and 114 is represented in Fig. 5. The financial impact from two scenarios transpiring together is a $85 \%$-trapezoidal fuzzy interval of $\{\$ 0.205 \mathrm{mln} ; \$ 0.548 \mathrm{mln} ; \$ 0.771 \mathrm{mln} ; \$ 1.692 \mathrm{mln}\}$, where an interval of $\{\$ 0.548 \mathrm{mln} ; \$ 0.771 \mathrm{mln}\}$ represents the range of the most probable financial impacts.

Applying similar logic, the financial impact of the entire set of available scenarios is summarized in a descending order of their alpha levels. The result of this describes a level of operation risk that exists in the organization for one in 100 years. In order to be compliant with Basel II requirements where the OR capital needs to be estimated with a precision of one worst year in 1000 years, the operation risk level estimates need to be adjusted by multiplying them by 10 .

In order to validate the proposed method for evaluation of operation risk, the real time experiment was undertaken. In a course of a year, different groups of banking experts from a particular banking organization were required to complete scenario workshops and come up with plausible storylines and estimates of extreme events that can potentially breach bank solvency and happen to their institution in a course of 100 years. The events had to cover all business activities and risk event types. Banking experts were informed by plausible internal and external OR loss data and supported by applicable media stories. In order to minimize bias of anchoring, the same training and facilitation were used in all scenario workshops. A set of 49 scenarios was derived. Application of the methodology described in this paper had delivered a fuzzy interval estimate (\$543mln, \$577.3mln, \$1,220mln, \$1,409mln). Alternative approaches deliver comparable estimates of the required OR regulatory capital: the Basic Indicator Approach computation resulted in $\$ 640 \mathrm{mln}$ and the Standardized Approach resulted in $\$ 1,080 \mathrm{mln}$.

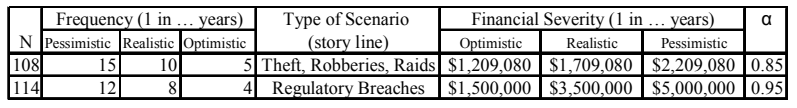

Table 1: Sample scenarios raw data

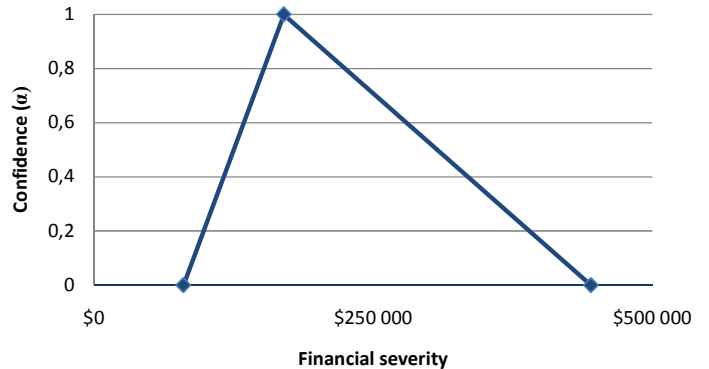

Fig. 3: Scenario 108 estimates corrected for 1-year horizon

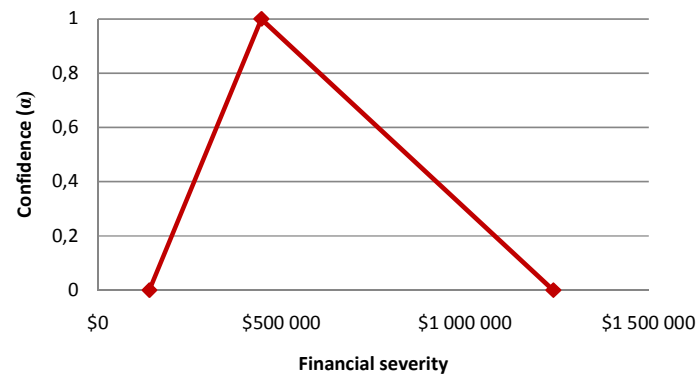

Fig. 4: Scenario 114 estimates corrected for 1-year horizon

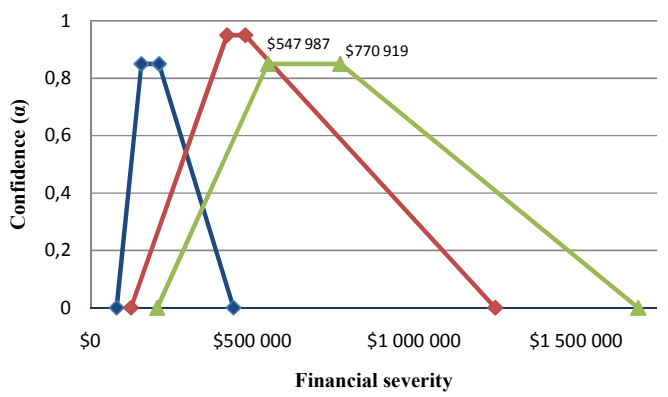

Fig. 5: Joint scenario estimates

\section{Conclusions and Future Research}

In contrast to the traditional approaches to compute precise probability of hypothetical scenario events using distribution or Bayesian approaches, this paper leverages on imprecise nature of scenario. It introduces and applies the methodology based on fuzzy probabilities and fuzzy severities of scenarios provided by experts to evaluate the level of operational risk and adequate capital required to cover it. We depart from severity and frequency triangular fuzzy numbers with their confidence assessment captured in alpha level. The contribution of the research is in the way trapezoidal fuzzy intervals of various heights are combined. The applicability of the proposed methodology is confirmed by a real-world experiment where operational risk exposure was computed using the current method, and more established approaches.

One can argue about the limitation of the proposed methodology. As one can notice, the operational risk level computed using the current methodology depends on the completeness and accuracy of the set of scenarios at hand. Complementary methods, possibly with the use of text mining technology, should be developed 
to frame unbiased set of potential storylines to be evaluated by experts. Future work needs to include research on interrelation between scenarios (conditional fuzzy probability), applicability of linguistic variables in describing scenario estimates instead of dollar amounts, development of a text mining classifier to group similar scenarios, assessing risk preferences of individual expert to limit bias, etc.

\section{References}

[1] Ackoff, R.L., Management Misinformation Systems, Management Science, 14, 1967, pp. 147-156.

[2] Alexander, C., Bayesians, Methods for Measuring Operational Risk, Derivatives Use, Trading \& Regulation, Vol. 6, No. 2, 2000, pp. 166-186.

[3] Basel II: International Convergence of Capital Measurement and Capital Standards: A Revised Framework - Comprehensive Version, Basel, June 2006.

[4] Buckley, J.J., Fuzzy Probability: New Approach and Applications, Springer-Verlag, 2005.

[5] Dubois, D., and H. Prade, Possibility Theory: An Approach to Computerized Processing of Uncertainty. Plenum Press, New York, 1988.

[6] Dutta, K., and Babel, D., Scenario Analysis in the Measurement of Operational Risk Capital: A Change of Measure Approach, Warton School of Business, University of Pennsylvania, Working paper \#101, March 2009.

[7] Heijden, K., R. Bradfield, G. Burt, G. Cairns, and G. Wright, The Sixth Sense: Accelerating Organizational Learning with Scenarios, Wiley, Chichester, 2002.

[8] Holton, G., Subjective Value-at-Risk, Financial Engineering News, 1 (1), 1997, pp. 1, 8-9, 11.

[9] Jungerman, H., and Thüring, M., The Use of Mental Models for Generating Scenarios, in: G. Wright, P. Ayton (Eds.), Judgmental Forecasting, Wiley, Chichester, 1987, pp. 245-266.

[10] Kahn, H., and A. J. Wiener, The Year 2000: $A$ Framework for Speculation on the Next Thirty-three Years, Macmillan, New York, New York, USA, 1967 , p. 6.

[11] Kaufmann, A., and M.M. Gupta, Introduction to Fuzzy Arithmetic: Theory and Applications. Van Nostrand Reinhold, New York, 1985.

[12] Kuchta, D., Fuzzy Capital Budgeting, Fuzzy Sets and Systems, 111, 2000, pp. 367-385.

[13]Lee, K.H., First Course on Fuzzy Theory and Applications, Springer-Verlag Berlin Heidelberg, 2005.

[14]Leon, C., and Reveiz, A., Operation Risk Management using a Fuzzy Logic Inference System, Borradores de Economia, 574, 2009.

[15] Mahmoodzadeh, S., Shahrabi, J., Pariazar, M., and Zaeri, M. S., Project Selection by Using Fuzzy AHP and TOPSIS Technique, International Journal of Human and Social Sciences, 1, 2007.

[16] Observed Range of Practice in Key Elements of Advanced Measurement Approaches (AMA), Basel, July 2009.
[17]Panjer, H., Operation Risk Modeling Analytics, Wiley Series in Probability and Statistics, John Wiley and Sons, 2006.

[18] Postma, T. J.B.M., and Liebl, F., How to Improve Scenario Analysis as a Strategic Management Tool?, Technological Forecasting \& Social Change, 72, 2005, pp. 161-173.

[19] Ringland, G., Scenario Planning: Managing for the Future, Wiley, Chichester, 1998.

[20] Schwartz, P., The Art of the Long View: Planning for the Future in an Uncertain World, Doubleday Currency, New York, 1991.

[21] Shah, S., Measuring Operational Risks using Fuzzy Logic Modeling, Towers Perrin-Tillinghast, July 2003

[22] Shevchenko, P. V., and Wüthrich, M. V., The Structural Modelling of Operational Risk via Bayesian Inference: Combining Loss Data with Expert Opinion, Journal of Operational Risk, 1(3), 2006, pp. 3-26.

[23] Smithson, C., and Song, P., Quantifying Operation Risk, Risk, 17, July 2004.

[24] Swart, R.J., Raskin, P., and Robinson, J., The Problem of the Future: Sustainability Science and Scenario Analysis, Global Environmental Change, 14(2), 2004, pp. 137-146.

[25] Taylor, D., Shelton, M., and Farmer, D., The Commonwealth Bank's Approach to Scenario Analysis, Operational Risk Scenario Analysis Workshop, July 2006.

[26] Wack, P., Scenarios: Unchartered Waters Ahead, Harvard Business Review, 63(5), 1985, pp. 73-89.

[27]Zadeh, L., Fuzzy Sets, Information Control, Vol. 8, 1965, pp. 338-353. 